\title{
El juego como método de aprendizaje
}

PLAY AS A LEARNING METHOD

○ JOGO COMO MÉTODO DE APRENDIZAGEM

Cristina Mora* / chiquis_moro@hotmail.com

Fanny Plazas** / faplagu@hotmail.com

Alcira Ortiz ${ }^{* * *}$ / xfpa2008@hotmail.com

Gladys Camargo*** / gladyshunza@gmail.com

\section{Resumen}

El objetivo general de la presente innovación es reconocer el juego como un referente metodológico. A partir de este objetivo se desprenden otros como identificar las diversas concepciones que se tienen del juego y sus clases: juego de roles, juego libre y lúdica; así como el valor educativo que se la ha dado en diferentes contextos.

Otro objetivo es innovar las prácticas pedagógicas a través del juego, en el Colegio Distrital Hunza, con el fin de generar aprendizajes verdaderamente significativos en los estudiantes.

Para lograr estos propósitos, las docentes innovadoras realizaron diversos proyectos de aula, y con el fin de dar rigurosidad a la innovación las docentes se valieron de referentes como: Juego, Método, Aprendizaje, Lúdica, Innovación y otros propuestos por teóricos que han abordado el tema, estableciendo una relación directa entre dichos referentes y las prácticas innovadoras que tienen las docentes en su quehacer pedagógico.

\section{Abstract}

The overall objective of this innovation is to recognize play as a methodological reference. There are other purposes derived from this overall objective, such as to identify different concepts about the play and its types, for example role play and free and fun play; as well as the educational value given to it in different contexts.

Another goal is to innovate pedagogical practices through play, in the Colegio Distrital Hunza, in order to generate truly meaningful learning in students.

To achieve these goals, innovative teachers carried out diverse classroom projects and, in order to give rigor to the innovation, teachers used references such as play, method, learning, fun, innovation, and other concepts proposed by theorists who have addressed the topic, seeking to establish a direct relationship between these references and the innovative practices these teachers implemented in their pedagogical activities.

\section{Palabras clave}

Juego, método, aprendizaje, lúdica, innovación.

\section{Keywords}

Play, method, learning, fun, innovation.

\footnotetext{
- Licenciada en Educación Preescolar, especialista en Didáctica y Pedagogía. Maestra del grado Preescolar.

* Licenciada en Educación Preescolar, Magíster en Administración y Supervisión Educativa. Maestra del grado Preescolar.

*.* Licenciada en Educación Preescolar, maestra del grado Preescolar.

**** Licenciada en Gestión y Desarrollo Pedagógico de la Educación Preescolar, Magíster en Educación.
}

Fecha de recepción: 27 de mayo de 2016 / Fecha de aprobación: 20 de junio de 2016 
O objetivo geral da presente inovação é reconhecer o jogo como uma referência metodológica. A partir desse objetivo, desenvolveram-se outros como identificar diversas concepções existentes sobre o jogo e suas classes: jogo de roles, jogo livre e lúdica; assim como o valor educativo que têm em diferentes contextos. Outro objetivo é inovar as práticas pedagógicas através do jogo no Colégio Distrital Hunza, visando gerar aprendizagens verdadeiramente significativas nos estudantes.

Para alcançar esses propósitos, as professoras inovadoras fizeram vários projetos de aula, e com o fim de dar rigorosidade à inovação, as docentes utilizaram referências como: Jogo, Método, Aprendizagem, Lúdica, Inovação e outras propostas por teóricos que já abordaram o tema, estabelecendo, assim, uma relação direta entre essas referências e as práticas inovadoras das docentes em sua labor pedagógica.

\section{Palavras chave}

Jogo, Método, Aprendizagem, Lúdica, Inovação
(...) El juego no sólo es aprendizaje de tal o cual técnica, de tal o cual aptitud, de tal o cual saber-hacer. El juego es un aprendizaje de la naturaleza misma de la vida que está en juego con el azar, con el alea.

Edgar Morin

\section{Y del problema, ¿qué?}

El punto de partida del presente proyecto de innovación educativa es la necesidad de cambiar y transformar las prácticas pedagógicas que se han venido desarrollando, tradicionalmente, en nuestras aulas. Recurrir al juego como método de la práctica pedagógica es la razón de la presente propuesta, ya que de manera tradicional el juego en la vida escolar se utiliza solo en las horas de recreo.

Siendo el juego un principio fundamental de la educación preescolar, son pocas las oportunidades en las cuales se utiliza en el aula de clase. Por lo general, se da prioridad al desarrollo de guías y a las actividades encaminadas a desarrollar la coordinación motriz fina, negando la oportunidad de realizar acciones diferentes. Parece sorprendente, pero el juego se relega de las aulas de preescolar, razón por la cual este solo tiene cabida en el momento de descanso. De esta manera, se desconoce la importancia que tiene el juego en la vida de los niños de preescolar.

Lo anterior se constituye en el motivo por el cual las docentes de los grados preescolares del Colegio Distrital Hunzá sedes B y C, jornadas mañana y tarde, se plantean el siguiente interrogante:
¿Cómo optimizar y utilizar el juego como método de aprendizaje, de manera que se constituya en una práctica pedagógica de gran significación para la vida de los niños y niñas?

\section{Así, se vive y convive}

El proyecto de innovación se desarrolla en un contexto educativo de la ciudad de Bogotá, en la localidad 11 (Suba), en el Colegio Distrital Hunzá. La comunidad educativa de esta institución pertenece a los estratos socio económicos 1 y 2 . Es una población fluctuante, ya que proviene de muchas familias desplazadas, se caracteriza por tener familias nucleares y recompuestas. Los padres de familia trabajan realizando diversos oficios, razón por la cual, suelen dejar a sus hijos solos, o al cuidado de terceras personas. Por lo tanto, la población infantil evidencia, en muchos casos, abandono. Estos hechos hacen que el colegio se constituya en el lugar donde muchos de los niños encuentran sustento alimenticio, cuidado y protección, así como actividades recreativas y nuevos aprendizajes. En esta dinámica, el juego desempeña un papel fundamental pues transforma los ambientes de aprendizaje y se constituye en el interés particular del presente proyecto de innovación.

Existen seis grupos de preescolar: tres en la mañana y tres en la tarde. Las edades de sus estudiantes oscilan entre los 4 y los 6 años. El colegio no cuenta con espacios suficientes para la recreación de estos niños, por lo que es necesario acudir a sitios comunitarios como son los parques, casa de la cultura y canchas de la zona. 


\section{El porqué del juego}

Juego: "Una actividad auto formadora de la personalidad del niño".

Jean Piaget

En el aula de clase suceden los grandes acontecimientos para la vida de nuestros estudiantes, acontecimientos que los marcarán para siempre. Allí, el juego toma gran relevancia para todos los aprendizajes, puesto que en la etapa de preescolar este se constituye en la forma de vida de los estudiantes, por consiguiente, en un instrumento poderoso por medio del cual el niño comienza a tener experiencias sociales fuera de su hogar y a interactuar con sus pares y maestros. Estas apreciaciones son las que han Ilevado a las docentes de Preescolar del Colegio Distrital Hunzá a implementar proyectos de aula que tengan como eje metodológico el juego.

Desde luego, la innovación propuesta tiene otros aspectos fundamentales, generados dentro la cotidianidad del aula, y que están estrechamente relacionados con el desarrollo social, cognitivo y motor de los niños y niñas. Ello, por supuesto, también motiva la presente innovación, en la medida en que ofrece a los lectores no solo la oportunidad de conocer dicha cotidianidad, sino, además, resaltar la importancia que tiene el juego en la vida escolar. Cuando se le da al juego una función formadora y no solamente se le erige como una actividad libre -como afirma Esteban Levin (2012) en el portal www.tvcrecer.com-, los chicos aprenden muchísimas cosas. Sin lugar a dudas, las actividades lúdicas ocupan la mayor parte del tiempo de los niños y a través de ellas se sientan los pilares de la educación.

De acuerdo con lo planteado, es imperativo volver a resaltar que el juego es la vida de los niños y las niñas en los primeros años. Se caracteriza el juego en esta etapa de la infancia por ser innato, libre, lleno de resultados fortuitos y de descubrimientos insospechados. Es, además, estímulo para la imaginación y, dada su propia naturaleza, de una enorme riqueza cultural. En este sentido, el juego es formador, siempre y cuando se afiance en el seguimiento de instrucciones y en el desarrollo de habilidades y capacidades, sustentadas tanto en el desarrollo como en el fortalecimiento de valores y normas. Es libre cuando los niños y niñas juegan con su cuerpo, con juguetes, manipulan objetos, y se mueven guiados por su propio sentido de curiosidad. Allí no hay reglas ni límites, no es rígido, ellos crean con sus juguetes, inventan a través de imágenes, expresan sentimientos, hacen preguntas y representaciones a través de él. Si todas estas experiencias libres o guiadas se transforman en presentaciones significativas y conscientes para su vida, entonces, en ese momento, se darán aprendizajes sobre la realidad que viven, lo cual significa, en pocas palabras, que al jugar crean y aprenden.

Por esta razón, se ha creado la necesidad de innovar, de transformar, y de aprovechar los recursos y espacios que se tienen, utilizando el juego como un método y una nueva forma de aprendizaje para los docentes del colegio Distrital Hunzá. Un hecho que ha trascendido y ha transformado sus prácticas, de tal manera que los niños de Preescolar han tenido otras formas más dinámicas y propias de acceder al conocimiento.

\section{¿Qué se propone el proyecto?}

Entre otros propósitos, se busca:

- Resaltar la importancia del juego en la edad preescolar y la forma como se dinamizan las diferentes concepciones que se tienen acerca de este dentro de las prácticas pedagógicas de las docentes del Colegio Distrital Hunzá.

- Fomentar el desarrollo del juego en el contexto educativo e implementar actividades lúdicas e innovadoras a nivel perceptivo y motor, promoviendo la creatividad, la atención, la memoria, la imaginación, para favorecer la apropiación del conocimiento y la relación con el entorno.

- Utilizar el juego como un instrumento de comunicación y de socialización para estimular la cooperación entre pares, enfatizando en el juego de reglas como una forma de aprendizaje e interacción social y, de esta manera, favorecer el autocontrol, la responsabilidad y la libertad, así como facilitar el autoconocimiento y el desarrollo personal. 


\section{Los referentes}

Los niños aprenden mucho más jugando que estudiando, haciendo que mirando. El juego que hacen solos sin el control de los adultos es la forma cultural más alta que toca un niño.

\section{Francesco Tonucci}

El juego se puede abordar desde varias perspectivas: como una actividad ligada a situaciones imaginarias, como una actividad libre, como una actividad para potenciar la lógica y conocer la realidad, como una oportunidad de aprendizaje, entre otras. Se caracteriza por ser natural, creativo; maneja un espacio y un tiempo; es imaginario, es una experiencia cultural en la cual se realizan descubrimientos y se establecen normas. En la edad preescolar se caracteriza por cierta forma de animismo: el niño da vida a los objetos inanimados; es una etapa de creatividad asombrosa aprovechando todo lo que tienen a su alrededor.

Y son las y los docentes de Preescolar quienes ven el juego desde su saber, desde su experiencia, desde los ambientes pedagógicos que viven y desde sus concepciones y contextos. Razón por la cual este proyecto de innovación, adelantado en el Colegio Distrital Hunzá, ha buscado dar a conocer cómo el juego se puede utilizar como método de enseñanza-aprendizaje, pues puede lograr que las prácticas pedagógicas se enriquezcan, varíen y, lo que es fundamental, que a través de esta metodología los niños estén motivados, expectantes, y que los aprendizajes sean verdaderamente significativos.

Por otra parte, para implementar el proyecto, las docentes elaboraron un marco de referencia, que lo fundamentan de modo pertinente desde tres ejes. Se toma cada uno por separado para claridad de los lectores.

\section{El juego}

El juego es innato en el ser humano. Todos los seres han aprendido desde su nacimiento a relacionarse con su familia y el mundo exterior a través del juego. Existen diversas referencias de juego: alegría, diversión, recreación, relajación, entre otras. Etimológicamente proviene de las palabras latinas iocum y ludus-ludere, que significa diversión, chiste. En el contexto educativo se define así:

Actividad necesaria para los seres humanos teniendo suma importancia en la esfera social, puesto que permite ensayar ciertas conductas sociales; siendo, a su vez, una herramienta útil para adquirir y desarrollar capacidades intelectuales, motoras o afectivas. Todo ello se debe realizar de forma gustosa y placentera, sin sentir obligación de ningún tipo y con el tiempo y el espacio necesarios. (Temas para la educación, 2011, párr. 1).

No se puede desconocer que, en general, en el medio escolar se tiene claridad sobre la importancia del juego y lo mucho que aporta al proceso educativo, basta mencionar algunos de los procesos cognitivos que desarroIla: la observación, la atención, la concentración y la memoria. Además, el juego consolida la imaginación y la creatividad, sin embargo, en la gran mayoría de los casos los docentes prefieren el uso del tablero y guías de trabajo a la realización de actividades lúdicas, lo que casi siempre frustra o liquida la creatividad.

Ahora bien, en la etapa infantil el juego es una actividad que potencia en gran medida todos los aprendizajes. Por ello, esta etapa se constituye en una atmósfera propicia para el desarrollo psicosocial y comunicativo, que permite a los niños y niñas poner a prueba los conocimientos que van adquiriendo progresivamente a través de las interacciones con el medio.

Es esencial tener presente que no se trata del juego por jugar, es decir, el simple activismo y desgaste de energías; es el juego como método de enseñanza y aprendizaje, con una intención, con una planificación y con unos objetivos específicos. Desde esta mirada, se hace entonces necesario conocer los propósitos de los juegos libres, los dirigidos, y sus respectivas funciones e implicaciones.

En primer lugar se debe destacar que el juego libre no es una "pérdida" de tiempo para las niñas y los niños, ni es una manera de tenerlos ocupados. Por el contrario, es un modo de favorecer la madurez del pensamiento creativo, así como forma de incrementar sus capacidades motoras y cognitivas. Es de suma trascendencia reconocer que 
en estos espacios lúdicos los niños comprenden cómo funcionan las cosas, descubren que existen reglas, causas, efectos, y la necesidad de adaptarse a ciertas pautas si desean que los demás los incluyan en sus juegos. El juego libre se caracteriza por ser espontáneo y con significado para quien participa en él.

El juego dirigido o supervisado por un adulto debe desarrollar los procesos socioafectivos, pues a través de él se crea el puente entre el adulto y el niño. En este contexto la importancia de la dirección del adulto, para el caso la maestra de preescolar, radica en que el juego se convierte en un instrumento de aprendizaje y de desarrollo. Por ello, los y las maestras deben ser asertivos en la toma de decisiones, pues no todas las maneras de hacerlo son adecuadas. Los juegos dirigidos son importantes porque los niños tienen la oportunidad de interactuar con su maestra, y de esta forma, llevar una vida fusionada con ella y con sus compañeros.

También es primordial conocer las diversas maneras de dirigir los juegos. Por ejemplo, se da la forma autoritaria, en la cual la maestra decide todo lo que se debe hacer. También está la dirección bilateral, en la que la maestra observa e indaga. Existe la pseudo democrática, en donde la maestra deja que el niño se exprese, pero al final ella es quien toma decisiones. Por último, hay una forma óptima de realizar los juegos dirigidos: la maestra da el modelo de juego y deja que el niño o niña actúe libremente, de tal manera que se dé la oportunidad de desarrollar destrezas, prácticas y hábitos a través de la experiencia social que se está viviendo.

\section{Los teóricos}

A lo largo de la propuesta se da gran relevancia a la importancia que tiene el juego en la edad infantil, en particular, en la forma en que favorece los aprendizajes, primero en el entorno familiar, guiado por los padres o adultos; luego, en el contexto escolar, mediado por los maestros. Es por esta razón que los docentes de hoy deben enfrentar otro reto: ser investigadores del entorno en donde se encuentran las instituciones educativas, para conocer de primera mano las necesidades. De ahí la importancia de permitir vivir a plenitud el juego, en especial, durante la infancia cuando se dan los cimientos de toda la vida.
De otro lado, Emilia Ferreiro y Ana Teberosky se enfocan en la necesidad de que los niños enfrenten la lectura y la escritura de forma natural y lúdica. Además, las autoras sostienen que la escritura no debe asumirse como un producto escolar, sino como un objeto cultural resultado del esfuerzo colectivo de la humanidad (Blog El Lenguaje escrito, s f.).

De acuerdo con Gallo (2001), en Colombia Juan Carlos Negret (s.f.) fundamenta su propuesta en tres paradigmas: Piaget, con su teoría del constructivismo genético; Vigotsky, con sus aportes sobre el valor de la socialización del acto lingüístico y, especialmente, en Emilia Ferreiro y Ana Teberosky, con su trabajo clásico sobre los sistemas de escritura en el desarrollo del niño (1979). Estos tres paradigmas los plasmó en su Programa Letras; en palabras de Negret: "la escritura está hecha para contar historias y el niño nunca deja de contar. Tal como aprende a hablar, aprende a escribir" (citado por Gallo, 2011, párr. 3). Las autoras tomaron de estos postulados algunas de sus propuestas para Ilevarlas a la práctica con los estudiantes.

\section{La innovación pedagógica}

En el ámbito escolar, el aula de clase se constituye en un lugar infranqueable en donde se dan relaciones sociales y jerarquías particulares que solo allí se manejan. Los procesos pedagógicos no son homogéneos y están en relación directa con los conocimientos que allí se imparten. Es un lugar multicultural, de transmisión de ideas, de conocimientos, de experiencias de vida, de negociación que no necesariamente están relacionadas con el currículo escolar, por tanto, es un lugar heterogéneo y de transformación.

En la relación e intercambio de saberes que se establecen en las aulas, se da lugar a transformaciones para mejorar las condiciones sociales y de convivencia, apoyados en el conocimiento. Es allí en donde la investigación educativa entra a jugar un papel preponderante, como un pilar del conocimiento, de reflexión, de crítica, de resolución de problemas, de reconocimiento de la realidad que se vive en el interior de las aulas, en la cotidianidad. Por consiguiente, se establece allí una relación entre la teoría y la práctica. 
Estas experiencias de investigación, tomadas con responsabilidad, pueden transformar las prácticas pedagógicas desarrolladas en la escuela, al generar verdaderos cambios tanto en el ámbito social, como en lo emotivo y lo cognitivo. La investigación educativa se concibe como la mirada rigurosa que pretende construir, explicar o comprender el fenómeno educativo en tanto se convierte a su vez en un proceso de aprendizaje para quienes participan en ella, de tal modo que ese aprendizaje les permita considerar una acción informada y reflexiva sobre la propia práctica. La problematización que hace el docente de su quehacer de manera sistemática y crítica, en la búsqueda de explicaciones o interpretaciones del mismo, ha permitido la cualificación de su profesión, y, por tanto, el perfeccionamiento de su ejercicio (Salcedo, s.f.).

Es así, como a través de la investigación e innovación pedagógicas, se crean verdaderos ambientes de aprendizaje, en donde los niños y las niñas guiados por la docente, a través del juego y la lúdica, tienen la oportunidad de crear espacios y aprendizajes verdaderamente significativos. Allí interactúan en diferentes situaciones y ambientes tanto físicos como culturales.

\section{Todo comenzó así}

El proyecto se ha venido realizando desde hace tres años en las sedes B y C del Colegio Distrital Hunzá, con la participación de las docentes de preescolar de las jornadas mañana y tarde. Fue una idea de la profesora Gladys Camargo, quien, al llegar a la institución, quiso poner en práctica la experiencia que había conocido en el sector privado. Se inició con los 25 estudiantes del grupo 06 del preescolar de la jornada tarde, cuyas edades oscilaban entre los 5 y 6 años, y estaba bajo la dirección de la profesora Gladys en el año académico 2008. En ese entonces, era el único preescolar que funcionaba en esa jornada en la sede C. Al trabajar con ese grupo la profesora evidenció la ausencia de la lúdica en las prácticas pedagógicas y el poco trabajo que se realizaba a nivel psicomotor, tarea que se delegaba únicamente al docente de educación física.

El convencimiento de la importancia que tienen todos los aprendizajes en el preescolar a través de la lúdica, principalmente, y la necesidad de sembrar fueron los motores que impulsaron la búsqueda de innovaciones en esta sede. Aún hoy, aprovechando el espacio de la cancha aledaña, se realizan juegos al aire libre y dentro del aula, que tienen como objeto el desarrollo polimotor, juegos de atención, memoria, roles, cooperativismo, rondas, entre otros. De igual manera, se comenzó a poner en práctica una dinámica a la que se le tiene mucha prevención en la escuela y ante la cual se escuchan un sinnúmero de reparos: la inclusión de los padres de familia en los procesos de enseñanza y aprendizaje de sus hijos. Sin embargo, invitar a los padres a la escuela, a través de los talleres de padres, ha sido una experiencia maravillosa que ha dado la grata ocasión de ver cómo las familias se sienten complacidas al poder participar activamente en la formación de sus hijos desde la escuela.

Ante el cierre del grado preescolar en esta sede, la profesora se trasladó a la sede B en el año 2010. Allí también encuentra que los niños carecen de espacio para el juego libre. Tratándose de una maestra emprendedora, con una visión dinámica y activa, rápida y efectivamente se hacen intercambios de saberes y de experiencias con la docente compañera de grado de la sede, y se Ilega a consensos acerca de la importancia del juego y la necesidad de abrir espacios para la lúdica, con el fin de seguir allí con la misma dinámica que se tenía en la sede C. Se proponen proyectos lúdicos, así como continuar con los talleres de padres. Desde entonces, se despliega una dinámica conjunta a través de proyectos encaminados al desarrollo integral, teniendo siempre como base el juego para generar nuevos conocimientos, verdaderamente significativos.

\section{Referencias}

Alternativo, E. y Guarda, A. (2016). Se aprende más jugando que estudiando: entrevista a Francesco Tonucci, niñólogo (1/2). El Blog Alternativo. Recuperado el 16 de diciembre de 2016, de http://www.elblogalternativo.com/2009/08/02/se-aprendemas-jugando-que-estudiando-entrevista-a-francesco-tonuccininologo/

Ferreiro, E. y Teberosky, A. (1979). Los sistemas de escritura en el desarrollo del niño. México: Siglo xxı. Recuperado de: www. oei.es/historico/inicial/articulos/sistemas_escritura_desarroIlo_nino.pdf

Gallo, C. (2011, 20 de febrero). Existe otra manera de aprender a leer y escribir. Vida de Hoy. Recuperado el 29 de julio de 2013, de http://www.eltiempo.com/archivo/documento/ MAM-4406445 
El lenguaje escrito. Bibliografía de Ana Teberosky. Recuperado de http://ellenguajeescrito.blogspot.com.co/p/bibliografia-de-anateberosky.html

Levin, E. (2012). El valor del juego en la infancia. Recuperado de http://www.tvcrecer.com/2012/valor-del-juego-en-la-infancia/\#. UhAfBSLXfSw importancia del juego

Morin, E. (1982). Ciencia con conciencia. Olimpíada de la filosofía de la República Argentina. Recuperado de https://olimpiadadefilosofiaunt.files.wordpress.com/2012/02/morin-1982-cienciacon-conciencia.pdf
Negret, J. (s.f.). Programa Letras. Tomado de: http://programaletras. com/

Salcedo, R. (s.f.). La investigación en el aula: y la innovación pedagógica. Recuperado de http://www.banrepcultural.org/blaavirtual/ educacion/expedocen/expedocen8a.htm

Temas para la educación. (2011). Federación de enseñanza de cc.oo. de Andalucía. Recuperado el 15 de diciembre de 2016, de https://www.feandalucia.ccoo.es/docu/p5sd8764.pdf

\section{Diálogo del conocimiento}

\section{En el juego de la vida}

En mi casa he reunido juguetes pequeños y grandes, sin los cuales no podría vivir. Son mis propios juguetes. Los he juntado a través de toda mi vida con el científico propósito de entretenerme solo. El niño que no juega no es niño, pero el hombre que juega perdió para siempre el niño que vivía en él y que le hará mucha falta ...

Pablo Neruda

Nuestro pensamiento tiene el gusto por la clasificación. Ansía el orden, la separación, la categorización; ama la taxonomía. Identifica analizar con clasificar, con organizar, con dividir los hechos en conjuntos ligados por semejanzas que les son propias, o que corresponden más a atributos que se crean en el mismo pensar. Es como si indagar significara establecer similitudes antes que diferencias. Para comprender, nos ocupamos de conformar conjuntos de cosas, de animales, de minerales...

Este proceder no se da solo al analizar el mundo que nos rodea. También aparece cuando tratamos de entender lo humano. Se agrupan las sensaciones, las situaciones, las condiciones, las expresiones, bien sean estas culturales, colectivas, o bien correspondan a la intimidad y a la singularidad de lo individual.

Nos cuesta la complejidad, nos perdemos en ella. Tenemos la necesidad de hacer semejante lo que es disímil, de simplificar lo que es complejo. El filósofo René Descartes, padre del racionalismo de la modernidad, enseñó en el siglo XVI que para asegurar el entendimiento pleno toda investigación y todo análisis debe, antes que nada, dividir las dificultades en tantas partes como sea posible hasta llegar a los elementos más sencillos y comunes. Con ello hizo de la racionalidad un refugio para protegernos de nuestras incertidumbres, un camino para evadir nuestras dudas.

La necesidad de simplificar, de hacer sencillo lo que es múltiple y confuso nos lleva también a clasificar y a separar de forma tajante las etapas de nuestras vidas. Nos aferramos a los rangos de edad para definir y para determinar actitudes, comprender comportamientos, establecer tendencias en nuestro desarrollo como individuos. Añoramos establecer con claridad en cada una de las fases de la existencia retos, temores, expectativas, gustos, necesidades o manifestaciones específicas. Ansiamos definir con precisión lo que es propio de la infancia, de la juventud, de la adolescencia, de la pubertad, de la madurez, o de la vejez, como si fueran cajones en los que podemos agrupar a todos quienes tienen en común un determinado número de años.

A quienes escapan de los estrechos límites de las etapas del desarrollo los señalamos como anormales. Los calificamos como inmaduros o como retrasados, si es que conservan comportamientos y actitudes que no corresponden a aquello que, en teoría, ya debe estar superado; mientras, los denominamos precoces o adelantados si son capaces de actitudes y conductas que atribuimos a personas de mayor edad. 
Estas determinaciones tan tajantes y extremas no son más que consecuencias de la comprensión estrecha que se impone acerca del desarrollo cognitivo. Nos aferramos a la creencia en un crecimiento lineal, a un progreso inexorable en el que se van superando etapas de manera irremediable, sin siquiera tener la posibilidad de mirar atrás. Mientras proclamamos la complejidad del desarrollo personal, mientras que decimos reconocer la multiplicidad de factores que intervienen en el crecimiento y en el aprendizaje, actuamos como si se tratara de procesos homogéneos que no están determinados por las situaciones y las condiciones en que se da nuestra vida.

Sin embargo, nuestra comprensión termina siendo limitada ante la complejidad de la existencia. A medida que crecemos, se mantienen actitudes y comportamientos que creíamos superados, o que, en nuestra manía de dividir y separar, suponemos son exclusivos de otros momentos de la vida. En la madurez se conserva la infancia de múltiples maneras, y los niños proceden como adultos y desarrollan hábitos y pautas que creemos son propios de los más mayores.

En esta riqueza y esta complejidad del crecimiento y del desarrollo personal, los niños no son adultos pequeños, ni los adultos son niños a quienes se les ha robado la sonrisa que tiene quien juega. El juego no es más que un ejemplo de esta imbricación y esta relación compleja que se da entre las etapas de la vida. Nada es más importante y trascendente en la vida de un niño que jugar. Nada es más serio y enriquecedor que el juego. Al jugar se aprende y construye con otros. Jugando se convive, se comparte, se crean lazos de solidaridad, se vive que los actos tienen consecuencias en las que se pierde y se gana. Por ello, los adultos deberían jugar hasta la hora de su muerte y por eso el derecho a ser niño pasa por el derecho a jugar.

Rafael Pabón García

144 nodos y nudos / volumen 4 N. ${ }^{\circ} 40$ / enero - junio de 2016/ISSN: 0122-4328/pp. 137-144/ 